\title{
UNA ESPECIE NUEVA DE FURCRAEA (AGAVACEAE) DE COLOMBIA
}

\author{
Diego Giraldo-Cañas
}

Herbario Nacional Colombiano (COL), Instituto de Ciencias Naturales, Universidad Nacional de Colombia, Bogotá D. C., Colombia; dagiraldoc@unal.edu.co (autor corresponsal).

\begin{abstract}
Giraldo-Cañas, D. 2020. A new species of Furcraea (Agavaceae) from Colombia. Darwiniana, nueva serie 8(2): 499-508.

As a result of recent studies of South American Agavaceae, a new species of Furcraea is described. This species is endemic of a small area of the Andean Cordillera Oriental of Colombia and it belongs to Furcraea sect. Furcraea. The uses and popular names are documented for the new species. Based on IUCN criteria, the new species is categorized as "critically endangered" (CR). Thus, a total of five species of Furcraea are currently known in Colombia, F. abisaii Giraldo-Cañas, F. acaulis (Kunth) B. Ullrich, F. cabuya Trelease, F. foetida (L.) Haworth, and F. selloana K. Koch. A key for the Colombian species is included. The new species is more similar to F. hexapetala (Jacq.) Urb. and F. selloana K. Koch, and therefore, their features are given.
\end{abstract}

Keywords. Agavaceae; endangered plant species; Flora of Colombia; Neotropical flora; xeric areas.

Resumen. Giraldo-Cañas, D. 2020. Una especie nueva de Furcraea (Agavaceae) de Colombia. Darwiniana, nueva serie 8(2): 499-508.

Como resultado de recientes estudios en Agavaceae sudamericanas, se describe una especie nueva de Furcraea. Esta especie es endémica de una pequeña área de la cordillera Oriental andina de Colombia y pertenece a Furcraea sect. Furcraea. Se documentan sus usos y sus nombres populares. De acuerdo con los lineamientos de la UICN, la nueva especie se ubica en la categoría "en peligro crítico" (CR). Así, el género Furcraea queda representado en la flora de Colombia por cinco especies, F. abisaii GiraldoCañas, F. acaulis (Kunth) B. Ullrich, F. cabuya Trelease, F. foetida (L.) Haworth y F. selloana K. Koch. Se presenta una clave para reconocer las especies presentes en Colombia. La nueva especie es más cercana a F. hexapetala (Jacq.) Urb. y F. selloana K. Koch, cuyas características se presentan.

Palabras claves. Agavaceae; áreas xéricas; especies vegetales en peligro; Flora de Colombia; flora neotropical.

\section{INTRODUCCIÓN}

Furcraea Vent., es un género neotropical, el cual se distribuye desde el centro de México hasta Paraguay, así como en las Antillas (Thiede, 2020). Este género se caracteriza por sus hojas espiraladas, inermes a más frecuentemente dentadas en sus márgenes, suculentas a semisuculentas, dispuestas en rosetas; inflorescencia terminal paniculada de grandes dimensiones (de hasta $15 \mathrm{~m}$ de alto), en la cual se disponen numerosas flores péndulas, perfectas, epíginas, trímeras, campaniformes, cortamente pediceladas, solitarias a más frecuentemente fasciculadas, de ovario ínfero y seis tépalos subiguales, agrupados en dos series, así como seis estambres insertos $c a$. $1 / 2$ la longitud de los tépalos, los cuales tienen los filamentos conspicuamente engrosados en su porción proximal y además, el estigma es trilobado, con estilo papiloso, engrosado proximalmente y triquetro; 
sus frutos son cápsulas trivalvadas, leñosas, cortamente estipitadas, rostradas y varían de oblongas o subglobosas a ovoides, en las cuales se disponen numerosas semillas en dos series por lóculo; las semillas son aplanadas, aladas, negras y brillantes (García-Mendoza, 2000; 2001; Thiede, 2020). Por otra parte, las inflorescencias presentan eventos de proliferación vegetativa (falsa viviparía), produciendo numerosos bulbilos, los cuales pueden ser bracteados o foliosos y constituyen una parte importante para la propagación de las especies, ya que éstos, una vez caen al suelo, pueden enraizar fácilmente y constituir nuevas rosetas (GarcíaMendoza, 2000; 2001; Thiede, 2020).

Sus especies pueden ser arborescentes (tallos de más de $1,5 \mathrm{~m}$ de alto) a más comúnmente subcaulescentes, generalmente restringidas a hábitats secos y sustratos pobres en nutrientes, entre el nivel del mar y los $3000 \mathrm{~m}$ de altitud (GarcíaMendoza, 2000; 2001; Thiede, 2020). A pesar de ser un género morfológicamente bien delimitado, es taxonómicamente complejo (García-Mendoza, 2000), y en este sentido, Furcraea sigue siendo uno de los géneros menos entendidos de la familia (Eguiarte et al., 2000), del cual se conocen 23 especies y numerosos sinónimos (Thiede, 2020). Por otra parte, es necesario destacar su importancia económica, pues desde tiempos precolombinos, varias de sus especies se han y se siguen empleando en la producción de fibras duras a gran escala, una práctica muy arraigada y vigente, principalmente en Colombia (Pérez Mejía, 1964; Ullrich, 1992; Cadefique, 2006; Thiede, 2020). Aquí se describe una especie nueva de Furcraea de Colombia y así, se empieza a cubrir algunos vacíos en torno de la taxonomía, la nomenclatura, la biogeografía y los usos de las especies colombianas y sudamericanas de Agavaceae y, particularmente, de Furcraea.

\section{MATERIALES Y MÉTODOS}

El análisis morfológico y la descripción de la especie aquí tratada se llevaron a cabo mediante los métodos convencionales de la taxonomía y la sistemática biológicas (Lawrence, 1962; Fonnegra, 1989). Se siguió el concepto morfológico de especie, con base en los postulados expuestos en McDade (1995), Wiens \& Servedio(2000)y deQueiroz(2007).
Se revisó la literatura concerniente al género Furcraea, haciendo énfasis en las descripciones, las ilustraciones y los protólogos de todos los binomios de este género, tanto nativos como cultivados, así como el análisis de numerosos materiales tipo. Por su parte, se siguió a Nyffeler \& Eggli (2020) y Thiede \& Eggli (2020), para el reconocimiento y la circunscripción de la familia Agavaceae, así como su ubicación en el orden Asparagales. La circunscripción y la delimitación del género Furcraea están basadas en Thiede (2020). Se emplearon los términos, en cuanto a la naturaleza armada de las hojas, dentada y diente (véase GarcíaMendoza, 2000, 2001). Para referirse a los eventos de proliferación vegetativa de las inflorescencias (falsa viviparía), se emplea el término bulbilo y no bulbillo (véanse Arizaga \& Ezcurra, 1995; Font Quer, 2001). El término lanceolado para referirse a la forma de las hojas está basado en Font Quer (2001). Las medidas de las diferentes estructuras vegetativas corresponden exclusivamente a plantas en floración. El nombre $F$. selloana $\mathrm{K}$. Koch se escribe de acuerdo con Thiede (2020) y no como aparece en la amplia literatura, F. selloa K. Koch. La clave está basada en datos personales, así como en García-Mendoza \& Lott (1994), Álvarez de Zayas (1996), García-Mendoza (2001), Wilcox (2005) y Thiede (2020). La categoría de riesgo de extinción se estimó con base en los lineamientos de la UICN (2012) y Bachman et al. (2011).

\section{RESULTADOS Y DISCUSIÓN}

Furcraea abisaii Giraldo-Cañas, sp. nov. TIPO: Colombia. Cundinamarca. Cordillera Oriental andina, municipio de Chipaque, carretera Bogotá-Villavicencio, sector Munar, cerca del desvío a Une, a $9 \mathrm{~km}$ de la plaza principal de Chipaque en dirección hacia Cáqueza, pendientes pronunciadas $\left(45-70^{\circ}\right)$, sustratos rocosos, pedregosos y arenosos (posiblemente de origen coluvial), con capa orgánica delgada $\mathrm{y}$ sujetos a fuerte erosión, pastizales-rosetales xerofíticos con árboles y arbustos dispersos, $4^{\circ} 25^{\prime} 51^{\prime \prime} \mathrm{N}-74^{\circ} 00^{\prime} 24^{\prime \prime} \mathrm{O}$, ca. $2100 \mathrm{~m}$ alt., 22VIII-2018, D. Giraldo-Cañas \& S. D. EspinelGalván 6204 (holotipo: COL; isotipos: COL, HUA). Figs. 1-4. 

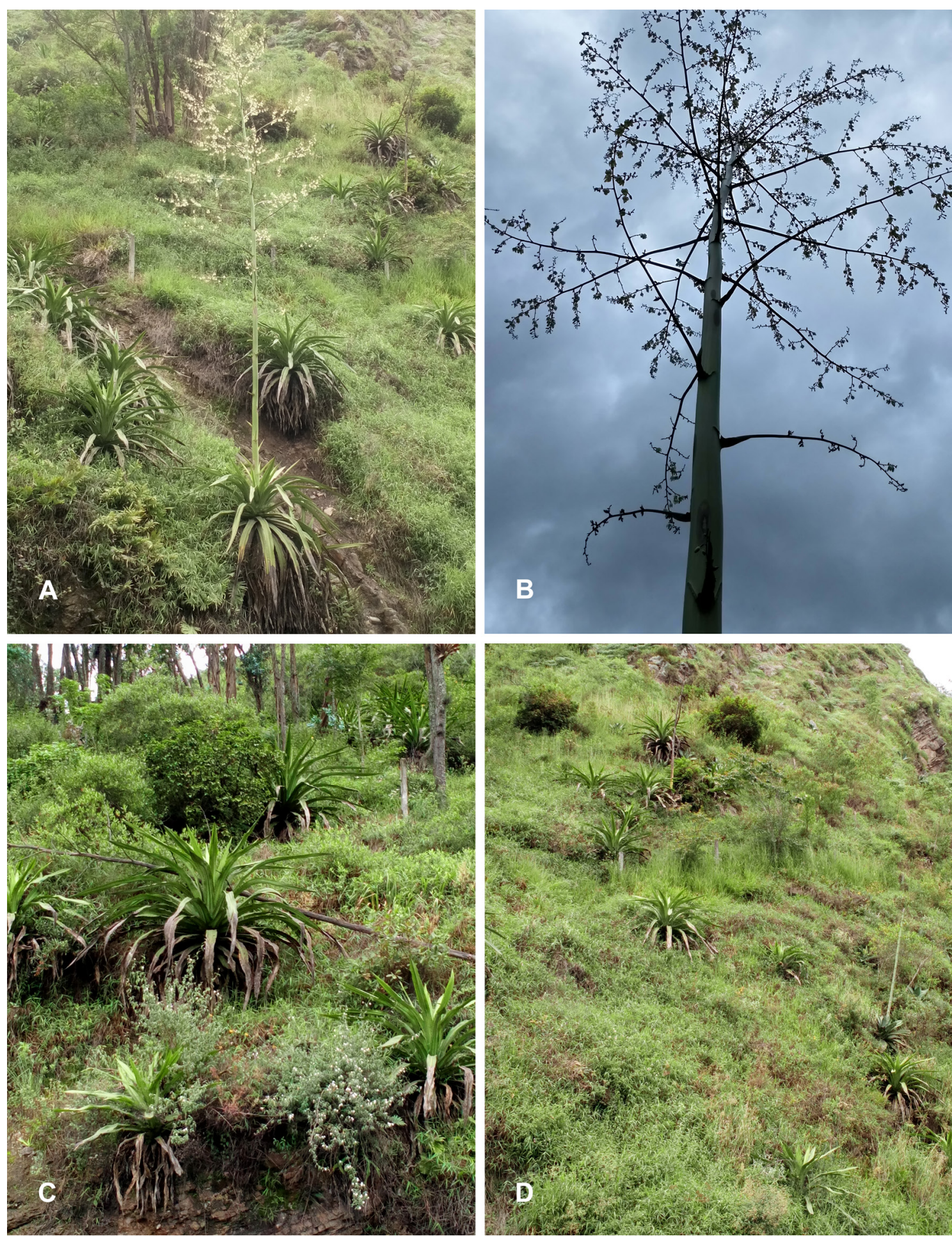

Fig. 1. Furcraea abisaii. A, roseta florífera. B, arquitectura de la panícula. C, D, hábitat. Todo de la población tipo. Fotografías: D. Giraldo-Cañas. Figura en color en la versión en línea http://www.ojs.darwin.edu.ar/index.php/ darwiniana/article/view/917/1196 
Diagnosis. Planta monocarpica, non-surculosa, subcaulescens, caule singulo, rosula densa, sphaerica, 3-4 m diam. Folia 160-200 × 10-12 cm, lineari-lanceolata, spina terminalis mucronata, $0.2-0.3 \mathrm{~cm}$ longa, margine denticulata, dentibus plerumque 0.2-0.4 cm longis, (0.5-) $1-3.5$ (-4) cm separatis curvatis. Inflorescentia scapo inclusa 6-7 $m$ alta, panicula pyramidali vel ovoidea, laxiflora, 25-34 ramis lateralibus 40-70 cm longis; bulbili bracteosi ovoidei; pedicellis glabris, brevibus, 0.4-0.6 cm longis, 1-3 in fasciculis. Flores $4.7-5.2 \mathrm{~cm}$ longis, tepalis elliptico-lanceolatis, $2.8-3.2 \mathrm{~cm}$ longis, glabris; filamenta $1.3-1.6 \mathrm{~cm}$ longa, papillosa, antherae $0.6-0.8 \mathrm{~cm}$ longa; gynoecium ovario leviter repandum, $1.5-1.9 \mathrm{~cm}$, stylus $1.5-1.8 \mathrm{~cm}$ longus, stigmati trilobato circa $0.15 \mathrm{~cm}$ diam. Capsulae et semina non vidi.

Roseta robusta, densa, esférica, 3-4 $\mathrm{m}$ de diámetro, hapaxántica, cortamente caulirrósula, tallo 0,44-0,47 $\mathrm{m}$ de alto y $22-24 \mathrm{~cm}$ de diámetro; sin hijuelos de ninguna clase (rizomatosos, infrafoliares, interfoliares). Hojas 160-200 × 10-12 cm, sésiles, espiraladas, gruesas, suculentasensuporción proximal, semisuculentas en sus porciones media y distal, coriáceas, fibrosas, rematando en un mucrón córneo, cónico, entero (sin canales y sin estrías), persistente, 0,2-0,3 cm long., éste proximalmente de color crema, a rojizo-granate distalmente, linear-lanceoladas, acuminadas, numerosas, (24-) 36-70 hojas por roseta, planas a cóncavas, recurvadas, verdes, opacas, con listones longitudinales verdes un poco más claros que el resto de la lámina, los listones de anchos diversos y más conspicuos en la cara adaxial, nerviación conspicua, constituyendo pliegues longitudinales muy gruesos; armadas con las márgenes dentadas, dientes numerosos, retrorsos a más frecuentemente antrorsos, simples, raramente algunos proximales bífidos, (0,2-) 0,3-0,4 cm long., distanciados entre sí (0,5-) 1-3,5 (-4) cm (los proximales más pequeños y más cercanos entre sí, mientras que los de las porciones media y distal más grandes y más separados entre sí), la base de los dientes granate, luego crema-amarillentos y en su extremo distal rojizos; la punta de los dientes se rompe fácilmente; las hojas senescentes persistentes en la porción proximal del tallo. Inflorescencia 6-7 m de alto, una panícula piramidal-ovoide, terminal, gigante, laxa, bracteada, bracteolada, bulbilífera con cientos de bulbilos por inflorescencia, éstos bracteados, ovoides, lisos, de color verde intenso brillante, fácilmente caedizos, 0,3-2,7 × 0,2-1,2 cm; pedúnculo florífero a partir del segundo tercio (el primer tercio sólo bracteado), $13-15 \mathrm{~cm}$ de diámetro en su porción proximal, verde claro y opaco, con corteza que se desprende en tiras; brácteas acuminadas, mucronadas, divergentes en ángulos ca. $45^{\circ}$ con respecto al eje de la inflorescencia;
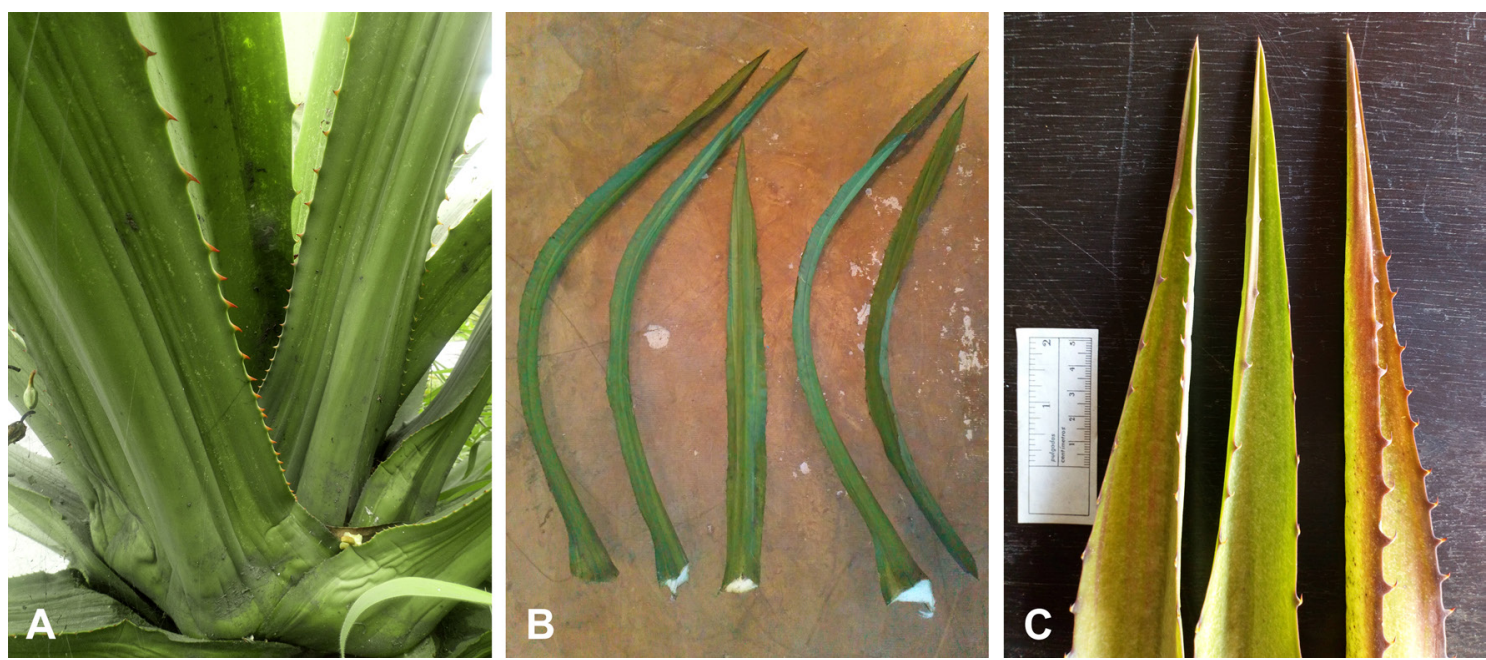

Fig. 2. Furcraea abisaii. A, porción proximal de una roseta florífera. B, hojas proximales de una roseta florífera. C, porción distal de las hojas. Todo de la población tipo. Fotografías: D. Giraldo-Cañas. Figura en color en la versión en línea http://www.ojs.darwin.edu.ar/index.php/darwiniana/article/view/917/1196 
brácteas proximales lanceoladas, dentadas (mucrón y dientes marginales), 40-90 $\times$ 4,5-9,7 cm; brácteas medias lanceoladas, dentadas (mucrón y dientes marginales), $10-40 \times 3-5 \mathrm{~cm}$; las brácteas proximales y medias con textura y colores similares a los de las hojas y con una ceñidura regular a ambos lados en su porción basal; brácteas distales deltoideatriangulares, inermes (sólo con un diminuto mucrón), negruzcas, quebradizas, 6-7 $\times 1-1,5 \mathrm{~cm}$; de cada bráctea surge una rama primaria horizontal, aunque en las brácteas más proximales dicha rama puede estar atrofiada o no se desarrolla; ramas primarias espiraladas, 25-34 por inflorescencia, separadas entre sí por 13-39 $\mathrm{cm}$; ramas primarias proximales 40-70 cm long., con 8-11 ramas secundarias cada una; ramas primarias medias 160-190 cm long., con 14-19 ramas secundarias cada una (ramas secundarias proximales de 7-20 $\mathrm{cm}$ long., ramas secundarias medias de $28-50 \mathrm{~cm}$ long., ramas secundarias distales de 20-35 cm long.); ramas primarias distales 9-40 cm long., sin ramas de orden consecutivo, muy raramente con 1-3 ramas secundarias de 4-8 cm long.; ramas secundarias espiraladas; bractéolas diminutas, deltoideas, papiráceas, fácilmente caducas, café claras a oscuras, en igual númeroquelas floresy basales aéstas. Floresperfectas, epíginas, trímeras, campaniformes, péndulas, (4,7-) 4,8-5,1 (-5,2) cm long., cortamente pediceladas (pedicelos glabros, verde-claros, 0,4-0,6 cm long.), numerosas, fácilmente caedizas, dispuestas en fascículos de 1-3 flores; tépalos 6, libres, en dos series, de igual longitud (los externos un poco más angostos que los internos), 2,8-3,2 × (0,6-) 0,8-1,2 cm, elípticolanceolados, glabros, cóncavos, en antesis opacos, carnosos, abaxialmente blanquecinos, adaxialmente verde claros con las porciones marginales escariosas y blanquecinas-translúcidas dispuestas a manera de un listón por margen (tépalos amarillentos cuando la flor se hace senescente); ovario ínfero, tricarpelar, trilocular, cilíndrico, ligeramente arqueado, $(1,5-) \quad 1,6-1,8 \quad(-1,9) \times 0,4-0,5 \mathrm{~cm}$, suculento, glabro, verde, opaco; óvulos anátropos, numerosos, dispuestos en dos series por lóculo; placentación axilar; estilo proximalmente engrosado, anguloso (ángulos redondeados y papilosos), triquetro, carnoso, 1,5-1,8 cm long., estigma trilobado, papiloso, $c a$. $0,15 \mathrm{~cm}$ de diámetro, estilo y estigma blanquecinos a verde muy claros; estambres 6, incluidos en la corola, filamentos engrosados en su porción proximal, subulados hacia su porción distal, papilosos, verde muy claros, 1,3-1,6 cm long., anteras introrsas, oblongas, dorsifijas, versátiles, de base sagitada y ápice emarginado, amarillas, 0,6-0,8 cm long.; botones florales opacos, verdes, con leves matices granates. Frutos desconocidos.

Etimología. La nueva especie está dedicada al Prof. Dr. Abisaí García-Mendoza (MEXU), un destacado botánico mexicano y experto en la familia Agavaceae, quien además, se caracteriza por su don de gentes, amabilidad y sencillez. Así, quiero rendirle con esta nueva especie, un sencillo homenaje y reconocimiento a su gran trayectoria académica e investigativa.

Nombres populares. Fique, penca (informantes: campesinos de la región).

Usos. Cercas vivas, combustible (los pedúnculos secos se emplean como leña), construcción (los pedúnculos se usan para construir enramadas rústicas - e.g. gallineros- y postes provisionales) (obs. pers.) y producción de fibras (no obstante, los campesinos de la región dicen que este último uso, ya no se da en la actualidad).

Distribución y hábitat. Esta nueva especie sólo se conoce de una pequeña región de xerofitia andina (vertiente orinocense de la cordillera Oriental; Dugand Gnecco, 1998) y hasta ahora sólo se ha documentado en algunas áreas contiguas de los municipios de Cáqueza, Chipaque, Choachí, Ubaque y Une (departamento de Cundinamarca), entre los 1800 y los $2200 \mathrm{~m}$ de altitud (Fig. 5), en donde constituye pequeñas subpoblaciones que no superan los cien individuos por subpoblación. Ésta crece en pendientes pronunciadas $\left(45-70^{\circ}\right)$, de sustratos rocosos, pedregosos y arenosos (posiblemente de origen coluvial), con capa orgánica delgada y sujetos a fuerte erosión, los cuales estaban caracterizados por pastizales-rosetales xerofíticos con árboles y arbustos dispersos (Baccharis macrantha Kunth, Clibadium cf. eggersii Hieron, Crotalaria sp., Croton pungens Jacq., Eucalyptus sp., Ficus americana Aubl., Piper aduncum L., Solanum dolichosepalum Bitter y Tecoma stans (L.) Kunth), mientras que los elementos herbáceos estaban dominados por Agave americana L. (naturalizada), Agave cundinamarcensis A. Berger, 
Agave sylvesteriana Giraldo-Cañas, Andropogon aequatoriensis Hitchc., Aristida laxa Cav., Cenchrus clandestinus (Chiov.) Morrone, Digitaria spp., Echeandia flavescens (Schult. \& Schult. f.) Cruden, Emilia fosbergii Nicolson, Euphorbia cyathophora Murray, Furcraea abisaii, Furcraea cabuya Trelease, Furcraea foetida (L.) Haworth, Ipomoea purpurea (L.) Roth, Melinis minutiflora P. Beauv., Pfaffia sp., Dicranopteris flexuosa (Schrad.) Underw., Pleopeltis macrocarpa (Willd.) Kaulf., Thelypteris patens (Sw.) Small, entre otras.

Conservación. Furcraea abisaii sólo se conoce de los enclaves xerófilos de una pequeña área (ca. $60 \mathrm{~km}^{2}$; extensión de presencia sensu UICN, 2012) de la Cordillera Oriental andina de Colombia (departamento de Cundinamarca), en donde constituye variassubpoblacionesdepocosindividuos. Dadas sus características de estrecha distribución geográfica, su reducido número de individuos, su distribución insular en medio de diversas matrices de xerofitia en diferentes estados sucesionales, las variadas y fuertes presiones a las que está sujeta, tanto antrópicas (obras civiles como la ampliación de la autopista al llano que fragmentó aún más las subpoblaciones y sacrificó numerosos individuos y la construcción de túneles y viaductos de grandes dimensiones, talas, quemas y establecimiento de cultivos y potreros para la ganadería, explotación de maderas y leña, construcción de zonas residenciales, fincas de recreo, entre otras), como naturales (suelos muy frágiles de pendientes pronunciadas y sustratos inestables, lluvias con torrenciales aguaceros, incendios forestales), y además, ninguna de las áreas donde crece esta especie tiene alguna categoría de protección, como parque nacional natural, reserva forestal o santuario de fauna y flora. Así, sobre la base de los criterios de la lista roja de la UICN (2012), esta especie debe ser ubicada en la categoría "en peligro crítico" (CR; B1ab(i, iii,iv)).

Comentarios. La nueva especie debe ser ubicada en Furcraea sect. Furcraea (véase Thiede, 2020: 324), ya que posee tallo corto, hojas mucronadas (mucrón córneo) y con márgenes conspicuamente dentadas, los dientes distanciados entre sí, así como bulbilos bracteados. Con el descubrimiento de esta nueva especie, se eleva a cinco el número de especies de Furcraea para Colombia (véanse Bernal \& Gradstein, 2016; Thiede, 2020) y a 24 el número de especies reconocido para el género. Por su parte, Bernal \& Gradstein (2016), citaron a F. stricta Jacobi para Colombia, pero no hay especímenes que respalden su presencia en este país, a pesar del amplio trabajo de campo realizado (Giraldo-Cañas, datos inéditos), por lo tanto, se excluye de este inventario. Las especies presentes en Colombia se pueden reconocer por medio de la siguiente clave.

\section{Clave para diferenciar las especies de Furcraea presentes en Colombia}

1. Hojas inermes, a lo sumo con muy pocos y diminutos dientes proximales; bulbilos foliosos ..... F. foetida (L.) Haworth 1. Hojas conspicuamente dentadas, dientes numerosos; bulbilos bracteados ................................................... 2

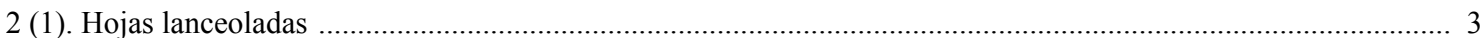

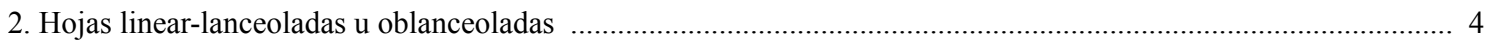

3 (2). Tallos 1-3,5 m de alto; hojas agudas; mucrón acanalado, 0,3-1 cm long.; bulbilos cónicos; flores geminadas; tépalos 0,20-0,23 cm long.; anteras 0,20-0,22 cm long.; ovario 1,7-2 cm long.; frutos subesféricos, 3,5-4 × 3-4 cm; semillas 0,12-0,15 cm long. F. acaulis (Kunth) B. Ullrich

3. Tallos de hasta 0,70 $\mathrm{m}$ de alto; hojas largamente acuminadas; mucrón cónico, 0,1-0,4 cm long.; bulbilos ovoides a ovoide-cónicos; flores solitarias o en fascículos de 2-6 flores; tépalos 0,25-0,32 cm long.; anteras 0,3-0,45 cm long.; ovario 2-2,8 cm long.; frutos oblongos, 5,5-7,5 × 3,5-4,5 cm.; semillas 0,15-0,17 cm long. F. cabuya Trelease 4 (2). Inflorescencias piramidales-ovoides, con 25-34 ramificaciones primarias; pedicelos 0,4-0,6 cm long.; tépalos elíptico-lanceolados, 2,8-3,2 cm long., porciones marginales escariosas; anteras 0,6-0,8 cm long.; ovario ligeramente arqueado; hojas linear-lanceoladas, recurvadas; mucrón persistente F. abisaii Giraldo-Cañas 4. Inflorescencias oblongas, con 40-60 ramificaciones primarias; pedicelos 0,5-1 cm long.; tépalos elípticos, 2-3 cm long., porciones marginales suculentas; anteras 0,30-0,32 cm long.; ovario recto; hojas oblanceoladas, erectas; mucrón caduco F. selloana K. Koch 

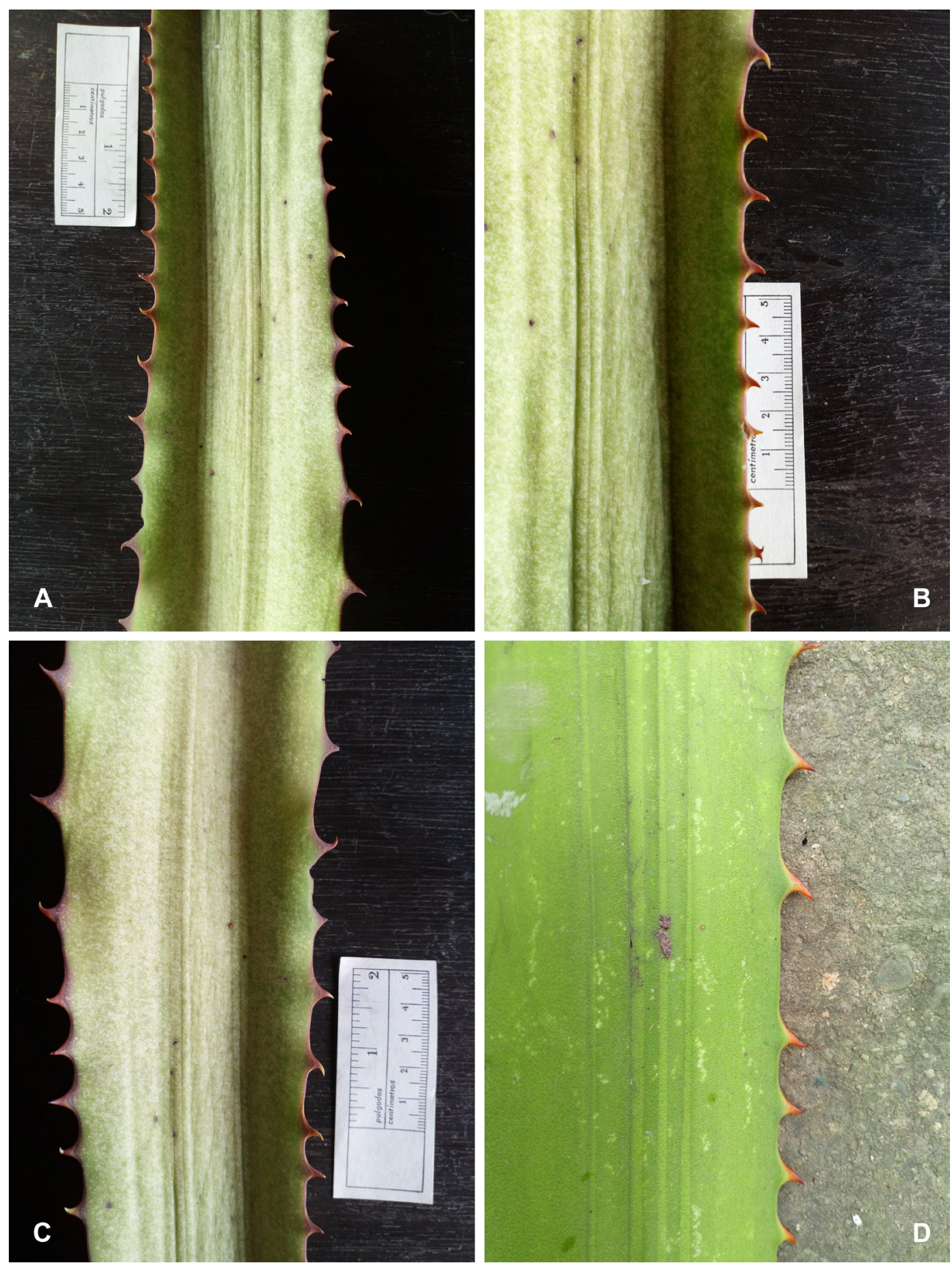

Fig. 3. Furcraea abisaii. A, dientes de la porción distal de una hoja. B, dientes de la porción proximal de una hoja (nótese un diente bífido). C, dientes de la porción media de una hoja (nótense los dientes, tanto retrorsos como antrorsos). D, porción media de una hoja. A, B y C corresponden a hojas de una roseta florífera, D corresponde a una hoja de una roseta no florífera; todo de la población tipo. Fotografías: D. Giraldo-Cañas. Figura en color en la versión en línea http:/www.ojs.darwin.edu.ar/index.php/darwiniana/article/view/917/1196 

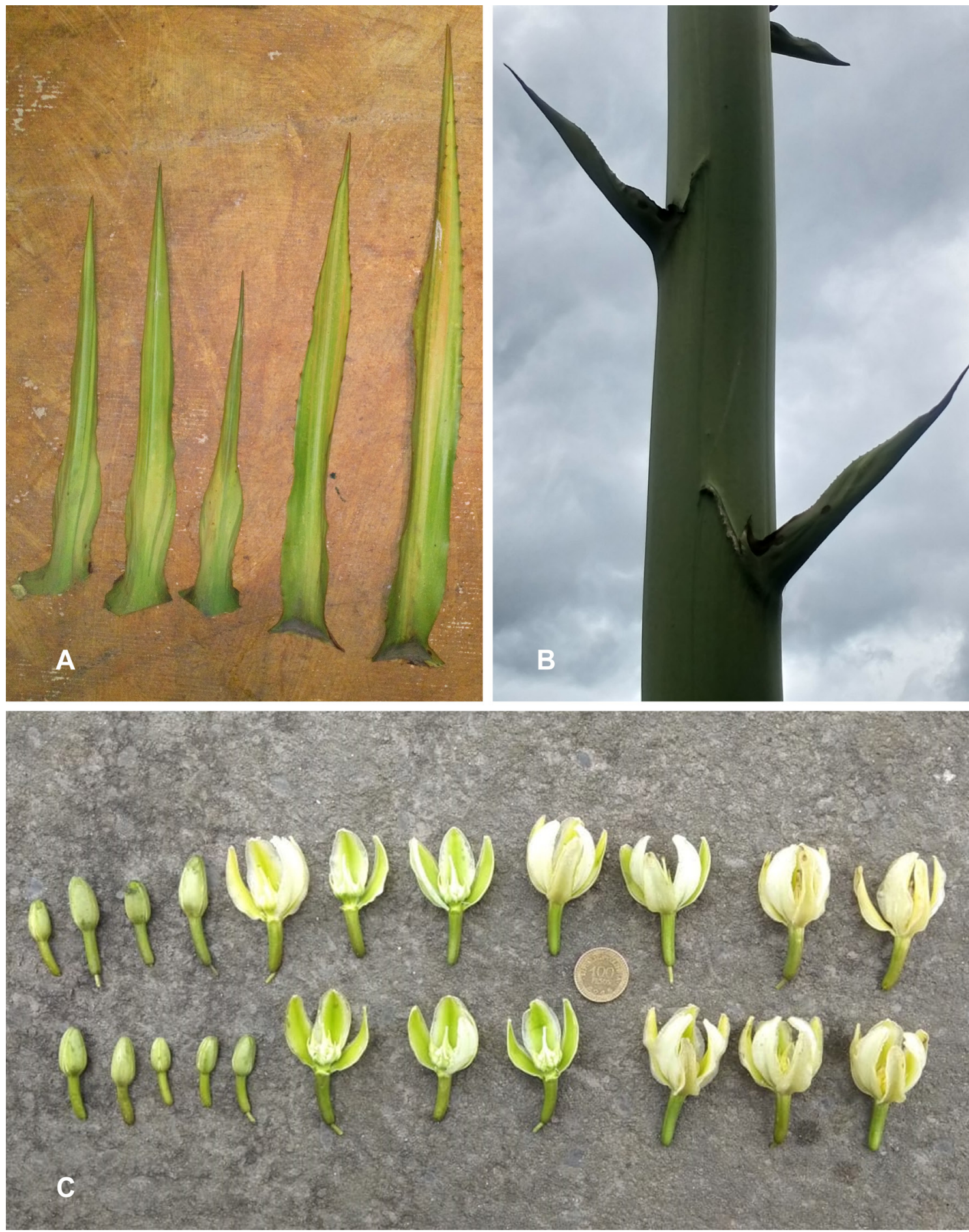

Fig. 4. Furcraea abisaii. A, brácteas proximales. B, brácteas de la porción media del pedúnculo. C, botones florales y flores. Todo de la población tipo; la moneda usada como escala mide $2 \mathrm{~cm}$ de diámetro. Fotografías: D. Giraldo-Cañas. Figura en color en la versión en línea http://www.ojs.darwin.edu.ar/index.php/darwiniana/article/ view/917/1196 


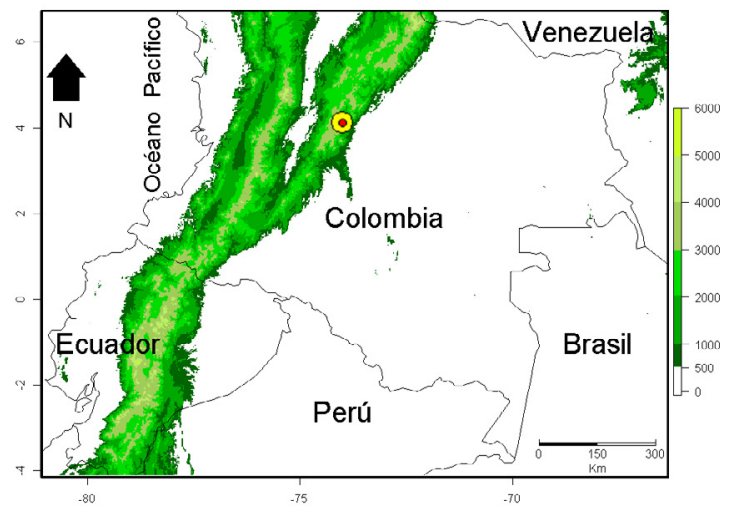

Fig. 5. Distribución de Furcraea abisaii, endémica de Colombia. La escala de colores representa la altura sobre el nivel del mar. Figura en color en la versión en línea http://www.ojs.darwin.edu.ar/index.php/darwiniana/ article/view/917/1196

Especies fenotípicamente similares. Furcraea abisaii es más similar a $F$. hexapetala (Jacq.) Urb. y $F$. selloana. De la primera especie, se separa por sus hojas lanceoladas y grandes dientes de $0,5-1,1$ cm long., los cuales están distanciados entre sí 3-10 $\mathrm{cm}$ (versus hojas linear-lanceoladas y pequeños dientes de 0,2-0,4 mm long., distanciados entre sí 0,5-4 cm en F. abisaii), por su mucrón acanalado (versus mucrón entero en $F$. abisaii), por su panícula romboidal (versus panícula piramidal-ovoide en $F$. abisaii), por sus tépalos elípticos de 2,5-3 cm long. (versus tépalos elípticos-lanceolados de 2,8-3,2 cm long. en F. abisaii), por sus filamentos más cortos de 1-1,3 cm long. (versus filamentos más largos de 1,3-1,6 cm long. en $F$. abisaii), y por sus anteras más cortas de 0,2-0,3 cm long. (versus anteras más largas de 0,6-0,8 cm long. en $F$. abisaii). De $F$. selloana, se distingue, principalmente, por sus hojas oblanceoladas y erectas (versus hojas linearlanceoladas y recurvadas en $F$. abisaii), por su mucrón caduco (versus mucrón persistente en $F$. abisaii), por sus inflorescencias oblongas (versus piramidales-ovoides en $F$. abisaii), por sus pedicelos más largos (versus pedicelos más cortos en $F$. abisaii), por sus tépalos elípticos más cortos (versus tépalos elípticos-lanceolados y más largos en $F$. abisaii), por sus anteras más cortas (versus anteras más largas en F. abisaii) y por su ovario recto (versus ovario ligeramente arqueado en $F$. abisaii).

\section{Paratipos}

COLOMBIA. Boyacá: Municipio de Villa de Leyva, vereda Salto y Lavandera, finca "Gatan asucune zрuуquyz quypcuas bzascua", a 5,7 km al noroeste de la plaza principal de Villa de Leyva, a 1,5 km de la intersección de la carretera veredal en el Alto de Los Migueles, en dirección al valle del río Cane, $2250 \mathrm{~m}$, 8-IX-2020, D. Giraldo-Cañas 6217 (COL, UPTC) (cultivada a partir de bulbilos obtenidos del ejemplar tipo). Cundinamarca: Cordillera Oriental andina, municipio de Ubaque, en arbustales de escarpes rocosos al borde de un camino veredal, $1955 \mathrm{~m}, 22-\mathrm{VIII}-2018, D$. Giraldo-Cañas \& S. D. Espinel-Galván 6205 (COL). Cordillera Oriental andina, municipio de Cáqueza, carretera Bogotá-Villavicencio, a $13 \mathrm{~km}$ de Chipaque, en laderas de xerofitia con pendientes pronunciadas en el borde de la carretera, sustratos rocosos, $1900 \mathrm{~m}, 22-$ VIII-2018, D. Giraldo-Cañas \& S. D. Espinel-Galván 6206 (COL). Cordillera Oriental andina, municipio de Choachí, camino veredal entre Choachí y la salida a la carretera Bogotá-Villavicencio, en pastizales secos degradados, 1825 m, 22-VIII-2018, D. Giraldo-Cañas \& S. D. Espinel-Galván 6207 (COL).

\section{AGRADECIMIENTOS}

Esta contribución es derivada de los proyectos "Estudios taxonómicos y morfológicos en los géneros Agave y Furcraea (Asparagaceae) en Sudamérica" (código Hermes 37417) y "Estudios florísticos en comunidades xerófilas de la Cordillera Oriental andina de Colombia" (código Hermes 26215), de la Universidad Nacional de Colombia, sede Bogotá D. C. A la Universidad Nacional de Colombia y a su Instituto de Ciencias Naturales, por todo el apoyo para la realización de las diferentes actividades de investigación. Al Dr. A. García-Mendoza por sus valiosos comentarios, grata colaboración y por el obsequio de preciada bibliografía. Al biól. M.Sc. Cristian Pinzón (COL) por el obsequio de muy variada y valiosa bibliografia. Al Dr. Guillermo Pino Infante (Jardín Botánico de Lima, Perú), por compartir bibliografía y por el regalo de varios individuos vivos de diferentes especies de Agave y Furcraea. Al Prof. Edwin Trujillo (Universidad de la Amazonia, Florencia, Colombia) y al ingeniero Óscar Perdomo (Universidade Federal do Rio Grande do Sul, Porto Alegre, Brasil), por la elaboración del mapa. A mis queridos amigos S. D. Espinel Galván, C. Pinzón Camacho, J. C. Camacho Bastidas, J. Sabagh y D. M. Díaz Rueda, por su apoyo en el trabajo de campo. Al Comité Editorial, así como a los evaluadores, por sus acertados comentarios. 


\section{BIBLIOGRAFÍA}

Álvarez de Zayas, A. 1996. El género Furcraea (Agavaceae) en Cuba. Anales del Instituto de Biología UNAM serie Botánica 67: 329-346.

Arizaga, S. \& E. Ezcurra. 1995. Insurance against reproductive failure in a semelparous plant: Bulbil formation in Agave macroacantha flowering stalks. Oecologia 101: 329-334. DOI: https://doi.org/10.1007/BF00328819

Bachman, S.; J. Moat, A. W. Hill, J. de la Torre \& B. Scott. 2011. Supporting Red List threat assessments with GeoCAT: geospatial conservation assessment tool. ZooKeys 150: 117126. DOI: https://doi.org/10.3897/zookeys.150.2109

Bernal, R. \& S. R. Gradstein. 2016. Asparagaceae. En R. Bernal, S. R. Gradstein \& M. Celis (eds.), Catálogo de plantas y líquenes de Colombia I, pp. 794-795. Bogotá D. C.; Universidad Nacional de Colombia.

Cadefique (Cadena Productiva Nacional del Fique). 2006. Guía ambiental del subsector fiquero. Bogotá D. C.: Ministerio de Ambiente-Ministerio de Agricultura.

de Queiroz, K. 2007. Species concepts and species delimitation. Systematic Biology 56: 879-886. DOI: https://doi. org/10.1080/10635150701701083

Dugand Gnecco, A. 1998. Geobotánica, botánica y ecología vegetal. Barranquilla: Corporación Autónoma Regional del Atlántico.

Eguiarte, L. E., V. Souza \& A. Silva-Montellano. 2000. Evolución de la familia Agavaceae: Filogenia, biología reproductiva y genética de poblaciones. Boletín de la Sociedad Botánica de México 66: 131-150. DOI: https://doi. org/10.17129/botsci.1618

Fonnegra, R. 1989. Taxonomía de las plantas vasculares. Medellín: Editorial Universidad de Antioquia.

Font Quer, P. 2001. Diccionario de botánica. Barcelona: Ediciones Península.

García-Mendoza, A. 2000. Revisión taxonómica de las especies arborescentes de Furcraea (Agavaceae) en México y Guatemala. Boletín de la Sociedad Botánica de México 66: 113-129.
García-Mendoza, A. J. 2001. Revisión del género Furcraea (Agavaceae). México D. F.: Facultad de Ciencias, Universidad Nacional Autónoma de México. Tesis doctoral inédita.

García-Mendoza, A. \& E. J. Lott. 1994. Furcraea Vent. Flora Mesoamericana 6: 45-47.

Lawrence, G. H. M. 1962. An introduction to plant taxonomy. Nueva York: The Macmillan Company.

McDade, L. A. 1995. Species concepts and problems in practice: insight from botanical monographs. Systematic Botany 20: 606-622. DOI: https://doi.org/10.2307/2419813

Nyffeler, R. \& U. Eggli. 2020. Introduction to the classification of Monocotyledons. En U. Eggli \& R. Nyffeler (eds.), Illustrated handbook of succulent plants. Monocotyledons, pp. 1-6. Berlín: Springer-Verlag GmbH Germany. DOI: https://doi.org/10.1007/978-3-662-56486-8

Pérez Mejía, J. A. 1964. El fique. Su taxonomía, cultivo y tecnología. Medellín: Compañía de Empaques-Editorial Colina.

Thiede, J. 2020. Furcraea-Agavaceae, en U. Eggli \& R. Nyffeler (eds.), Illustrated handbook of succulent plants. Monocotyledons, pp. 323-347. Berlín: Springer-Verlag GmbH Germany, part of Springer Nature. DOI: https://doi. org/10.1007/978-3-662-56486-8_106

Thiede, J. \& U. Eggli. 2020. Agavaceae, en U. Eggli \& R. Nyffeler (eds.), Illustrated handbook of succulent plants. Monocotyledons, pp. 9-19. Berlín: Springer-Verlag GmbH Germany. DOI: https://doi.org/10.1007/978-3-662-56486-8

UICN. 2012. Categorías y criterios de la lista roja de la Unión International para la Conservación de la Naturaleza UICN. Versión 3.1. Gland, Suiza. Segunda edición. https://www. iucn.org/es/content/categorias-y-criterios-de-la-lista-rojade-la-uicn-version-31-segunda-edicion

Ullrich, B. 1992. Furcraea (Agavaceae) en Sudamérica. Quepo 6: 67-75.

Wiens, J. J. \& M. R. Servedio. 2000. Species delimitation in systematics: inferring diagnostic differences between species. Proceedings of the Royal Society B 267: 631-636.

Wilcox, M. 2005. The genus Furcraea (Agavaceae) in the Auckland region. Journal of the Auckland Botanical Society. 60: 159-162. 